\title{
Heart rate variability as an autonomic biomarker in ischemic stroke
}

\author{
Variabilidade da frequência cardíaca como biomarcador autonômico em pacientes com \\ acidente vascular cerebral isquêmico
}

Natalia BUITRAGO-RICAURTE', Fátima CINTRA², Gisele Sampaio SILVA

\begin{abstract}
Stroke is one of the leading causes of mortality and disability worldwide. Autonomic dysfunction after ischemic stroke is frequently associated with cardiac complications and high mortality. The brain-heart axis is a good model for understanding autonomic interaction between the autonomic central network and the cardiovascular system. Heart rate variability (HRV) analysis is a non-invasive approach for understanding cardiac autonomic regulation. In stroke patients, HRV parameters are altered in the acute and chronic stages of the disease, having a prognostic value. In this literature review we summarize the main concepts about the autonomic nervous system and HRV as autonomic biomarkers in ischemic stroke.
\end{abstract}

Keywords: Heart Rate Determination; Autonomic Nervous System; Biomarkers; Stroke.

\section{RESUMO}

O acidente vascular cerebral (AVC) é uma das principais causas de mortalidade e deficiência no mundo inteiro. Alterações do sistema nervoso autônomo após AVC estão frequentemente associadas a complicações cardíacas e alta mortalidade. 0 eixo cérebro-coração é um modelo apropriado para entender as interações entre a rede central autonômica e o sistema cardiovascular. A análise da variabilidade da frequência cardíaca (VFC) é uma aproximação não invasiva para o entendimento da regulação autonômica cardíaca. Em doentes que sofreram um AVC, os parâmetros da VFC se encontram alterados nas fases aguda e crônica da doença, com a possibilidade de valor prognóstico. Nesta revisão da literatura, apresentamos os principais conceitos sobre o sistema nervoso autônomo e a variabilidade da frequência cardíaca como biomarcador autonômico no acidente vascular cerebral isquêmico.

Palavras-chave: Determinação da Frequência Cardíaca; Sistema Nervoso Autônomo; Biomarcadores; Acidente Vascular Cerebral.

Stroke is one of the leading causes of mortality and disability worldwide ${ }^{1}$. More than $40 \%$ of the patients with stroke have sequelae with long-term consequences. Despite the advent of new diagnostic and therapeutic technologies during the $21^{\text {st }}$ century, stroke continues to be a public health problem ${ }^{2}$. One explanation for this phenomenon is its multifactorial origin as well as the incomplete understanding of the etiopathological mechanisms underlying the natural history of the disease ${ }^{3}$. Alterations of the autonomic nervous system after ischemic stroke (IS) may play a role in stroke recurrence and recovery. The brain-heart axis is an appropriate model for understanding heart autonomic regulation and its interaction with the autonomic central network (ACN), that can be injured after stroke ${ }^{4,5,6}$.
Because of technical difficulties to access the autonomic function at the ACN due to multiple structures and widespread connections involved, approaches based on response measurements in the effector systems or organs are usually used ${ }^{7}$. The analysis of heart rate variability (HRV) is a noninvasive approach to the autonomic function that requires only an electrocardiographic record. With HRV it is possible to analyze in the time and frequency domains parameters associated with parasympathetic or sympathetic activity and its variations in different conditions. Changes in autonomic activity in stroke patients are well described in the acute phase related to cardiac and infectious complications that are the leading causes of nonneurological death ${ }^{8,9}$. Furthermore, it seems that the autonomic impairment persists during the chronic phase of

\footnotetext{
${ }^{1}$ Universidade Federal de São Paulo, Department of Neurology, São Paulo SP, Brazil.

${ }^{2}$ Universidade Federal de São Paulo, Department of Cardiology, São Paulo SP, Brazil.

Natalia BUITRAGO-RICAURTE (D) https://orcid.org/0000-0003-2894-2203; Fátima CINTRA (iD) https://orcid.org/0000-0002-3361-7061;

Gisele Sampaio SILVA (iD https://orcid.org/0000-0002-3247-3123

Correspondence: Natalia Buitrago-Ricaurte; E-mail: natibuitragori239@gmail.com

Conflict of interest: There is no conflict of interest to declare.

Authors' contributions: All authors contributed to the design and implementation of the search strategy and to the writing of the manuscript.

Received on January 14, 2020; Received in its final form April 4, 2020; Accepted on April 18, 2020.
} 
stroke, impacting the prognosis of patients. Therefore, there is a growing interest in HRV analysis as a potential tool for prognosis and understanding of underlying mechanisms in post-stroke autonomic dysfunction.

We searched the following databases for published studies in Portuguese, English and Spanish languages over the period 2000-2019: PubMed, Excerpta Medica Database (Embase), Scientific Electronic Library Online (SciELO) and ProQuest. The following terms were used to generate searches: stroke, ischemic stroke, heart rate variability, heart rate determination, heart rate, acute stroke, chronic stroke. The search process included articles that described stroke phase, HRV determination method and specific outcomes. In this literature review we present basic concepts about the autonomic nervous system, the principal approaches to study autonomic dysfunction in the clinical setting and HRV as a promising prognostic tool in IS patients.

\section{THE AUTONOMIC NERVOUS SYSTEM}

The autonomic nervous system (ANS) is an intricate, hierarchical, multifaceted neuronal circuit that regulates physiological homeostasis, by controlling different systems (cardiovascular, gastrointestinal, genitourinary, thermoregulatory, immune, etc.) in response to internal and external stimuli ${ }^{10}$. This system modulates the function of each organ in the human body by the innervation of smooth muscle, glands and cardiac muscle tissue. In an evolutionary perspective, the ANS is related to ancient evolutionary processes associated with organisms' adaptation for survival and exemplified in the human response to external injuries known as the fight-or-flight reaction ${ }^{11}$. But the development of the ANS concept is relatively recent and a product of dynamic interactions between history, science and social needs. Recently, autonomic alterations were described as highly prevalent in common diseases like hypertension, diabetes, Parkinson's, stroke and sleep disorders. However, because of the complexity and inaccessibility of the whole ANS for physiological testing, exploring the autonomic function remains an actual challenge.

\section{Structures and principal functions}

The autonomic nervous system is a complex organization that operates by the interaction of multiple circuits composed of afferent, integrating and efferent elements working together through visceral reflexes ${ }^{12}$. This is an unconscious process that starts with the communication of organ demands through neurotransmitters, hormones and electrolytes to an integrating center (reticular substance, tractus solitarius, hypothalamus, cortical structures) that processes the information and generates responses that will reach the target organ or systems. In order to do this, the system uses ways to communicate signals: preganglionic, postganglionic neurons and integrative ganglia.
The systems classically subdivided into parasympathetic and sympathetic function synergistically and independently to control effector organs with different innervation patterns ensuring their integrative intervention for suitable regulatory control ${ }^{10}$. In an oversimplistic manner, it can be said that the sympathetic division allows organisms to respond to threats, danger and stress. The parasympathetic is recognized by its role in conserving energy, controlling digestion and protecting the body against wastes. Besides having different functions, a macroscopic anatomic distribution in the parasympathetic system is made by a long preganglionic neuron located in the brainstem nuclei or at the sacral level of the spinal cord. The preganglionic sympathetic neurons are shorter and located in the intermediolateral cell column at the T1-L2 levels and activate neurons in the paravertebral, prevertebral ganglia and endocrine adrenomedullary cells (Figure 1). The two systems are not mutually excluding and other variables modulate responses as well as the efferent stimulus. The autonomic centers in the brainstem act as relay stations for the control of activities initiated in higher levels of the brain, defined as the autonomic central network. In addition, the ANS is not isolated and functions in conjunction with other regulatory systems, maintaining the organism's homeostasis, adding more variables to the ANS integrative model ${ }^{11}$.

\section{Autonomic central network}

The ACN is a conglomerate of interconnected brainstem and forebrain structures involved in the regulation of baseline functions of the autonomic nervous system. The $\mathrm{ACN}$ is an integrative center that modulates the responses of different physiological systems (respiratory, cardiac, vascular, digestive and endocrine) to integrate the internal and external stimuli in an adaptive way, optimizing the organism's metabolic resources ${ }^{4}$. The ACN is distributed throughout the neuraxis. Initially the main regions involved were: the insula, prefrontal medial cortex, amygdala, hypothalamus, periaqueductal gray matter, parabrachial nucleus and intermediate reticular zone in the medulla ${ }^{12}$. Like the central nervous system, the ACN functions in a hierarchical way and the cortical areas are involved in the high-order autonomic control. The central amygdaloid nucleus and the stria terminalis are structures related to emotion and its autonomic manifestations. The homeostasis and stress responses are modulated by the paraventricular, lateral and dorsomedial nucleus of the hypothalamus that activates endocrine, vasomotor and digestive functions. The brainstem nucleus (periaqueductal, parabrachial, tractus solitarius, intermediate reticular zone) are integrative centers that modulate viscerosensory, humoral and exteroceptive afferences with motor and autonomic effectors in vasomotor, cardiovagal and respiratory systems (Figure 2).

The ACN is a subsystem of the ANS and, as part of a complex organization regulating other physiological systems, 


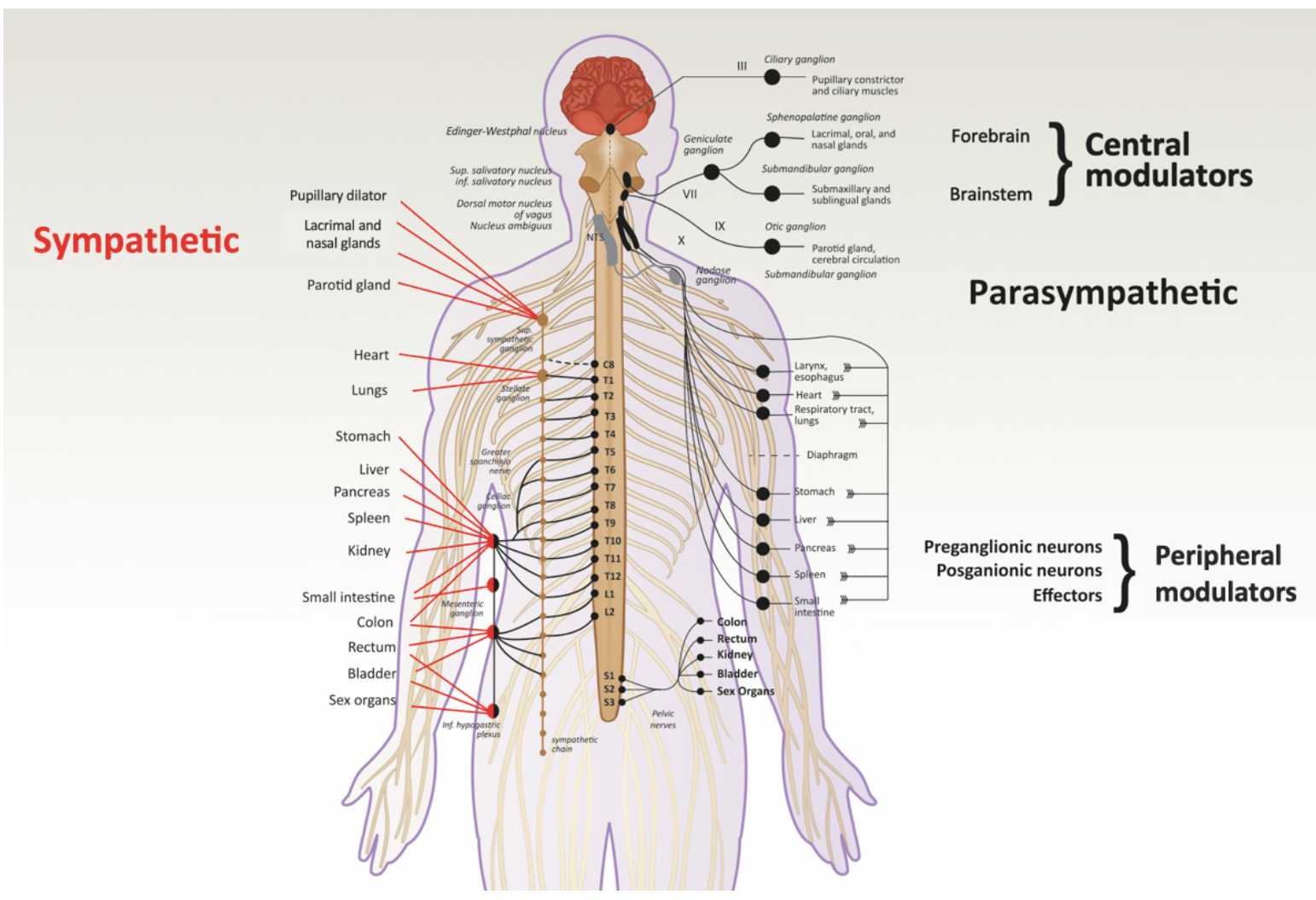

Source: adapted from Wehrwein et al.10.

Figure 1. The autonomic nervous system configuration. The autonomic nervous system sustains vegetative functions in different physiological systems to maintain the organism's life. For this purpose, it has a hierarchical and complex distribution with central and peripheral modulators. The central modulators are multiple forebrain and brainstem structures that receive, regulate and send impulses in an adaptive response manner. The peripheral modulators are classified as sympathetic and parasympathetic systems according to the anatomical distribution of the preganglionic and postganglionic neurons and to the responses observed in the target organ. Peripheral modulators are pathways between the central modulators and the effector systems.

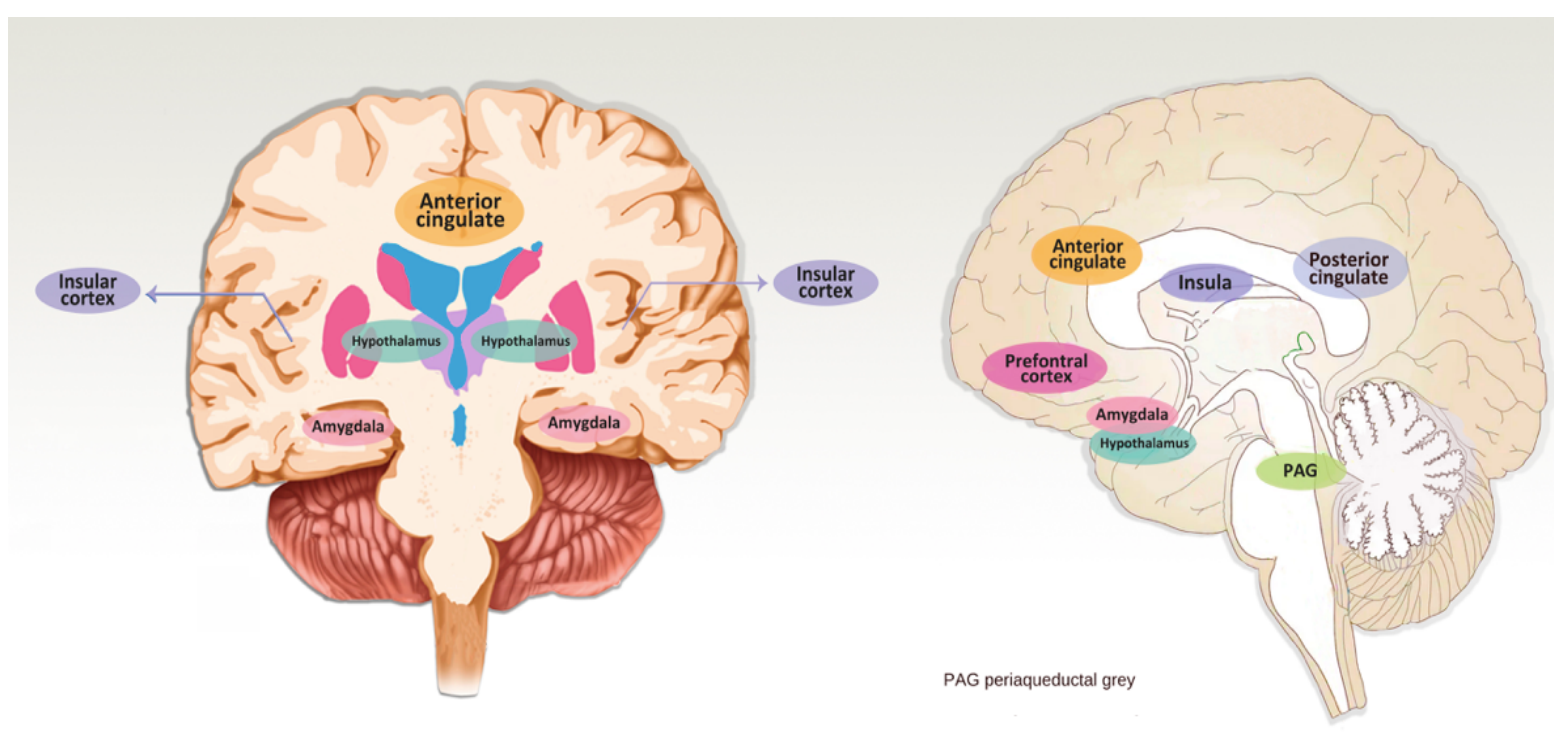

Source: the authors.

PAG: periaqueductal grey

Figure 2. The autonomic central network. The main structures involved in central autonomic control are the cingulate, insula, prefrontal cortex, amygdala, hypothalamus and brainstem structures (periaqueductal gray matter, solitary tract nucleus). The cortical structures are involved in high order autonomic control and are related to emotions, memory, stress and homeostatic responses. The brainstem structures are integrative centers modulating viscerosensory, humoral and exteroceptive afferents with motor and autonomic effectors in vasomotor, cardiovagal and respiratory systems. 
requires interconnections, hierarchical coordination, statedependent activity and neurochemical modulation ${ }^{13,14}$. The physiological functions of the ACN include tonic excitation to autonomic and respiratory motoneurons, coordination of spinal preganglionic structures, regulation of cardiovascular, respiratory and digestive autonomic responses and integration of afferent stimulus. The final objective of these coordinated processes is to create the most effective efferent responses in diverse systems, maintaining homeostasis according to the organism's required thresholds.

\section{Approaches to autonomic nervous system evaluation}

The ANS was classically subdivided as parasympathetic, sympathetic and enteric according to its effector functions. Recently, this strict classification has been questioned because the interaction of the subdivisions follows an integrative, synergic and bidirectional modulation ${ }^{15}$. In this complex scenario, there are also technical difficulties to access the autonomic function due to the involvement of multiple structures, regulators and widespread connections. In order to try to simplify this problem, current approaches to ANS evaluation are based on inducing autonomic reflexes and measuring responses in effector systems or organs.

These measures attempt to quantify autonomic responses giving specific values for sympathetic or parasympatheticrelated activities. Some methods described for testing the autonomic function in the clinical setting include: cardiovagal autonomic testing (HRV, heart rate - HR response to Valsalva maneuver, postural changes, isometric exercise and cold pressor test), vasomotor adrenergic autonomic testing (blood pressure response to Valsalva, head-up tilt table test), sudomotor testing (quantitative sudomotor axon reflex test - QSART, thermoregulatory sweat test - TST) ${ }^{7}$.

In some neurological diseases, these tests are mandatory for diagnostic and prognostic purposes. They can also be used for the evaluation of common conditions associated with autonomic dysfunction such as syncope, orthostatic hypotension, autonomic neuropathy, postural tachycardia syndrome and sweat alterations ${ }^{16}$. The composite autonomic symptom score 31 (COMPASS 31) could be used as a complementary tool to assess autonomic symptoms ${ }^{17,18}$.

\section{HEART RATE VARIABILITY: AN APPROACH FOR AUTONOMIC EVALUATION}

\section{History}

Certainly, since immemorial times, human beings have noticed changes in the beat-to-beat of their hearts. Variations with fear, physical activity, sexual arousal and rest are part of daily life. From a historical perspective, the study of pulse characteristics has been followed by quantitative evaluations of pulse and HR and finally methods that quantify changes in the electrical current of the heart, focusing on the study of beat-to-beat variation with advances in clinical applications ${ }^{19,20}$.

The concept of HRV as a method for studying the autonomic function was first described in 1965 by Hon and Lee to correlate fetal distress with changes in the $\mathrm{HR}^{21}$. Findings during the 1970s demonstrated an association of HRV and multiples diseases, such as autonomic neuropathy in diabetes, post-infarction mortality and altered autonomic function in patients with heart failure ${ }^{22}$. In the clinical setting, several studies attempting to use HRV as a diagnostic and prognostic tool demonstrated that nonlinear approaches are more valuable than linear methods. For example, one of the most relevant applications of HRV is as prediction tool for mortality in neonatal sepsis ${ }^{23,24}$. Currently it is known that variations in the HR are the product of intricate interactions between parasympathetic and sympathetic fibers and their effectors.

\section{Definition}

HRV refers to beat-by-beat heart variations as a result of a physiological variation in the duration of the RR intervals between sinus beats ${ }^{21,25}$. As a physiological response, HRV is the consequence of multiple processes that modulate the depolarization rate of the cardiac sinoatrial node (SAN), the major heart pacemaker. The HR is a result of the autonomic tone predominance, modulated by the SAN innervation by parasympathetic and, in less extent, sympathetic fibers. Also, humoral factors influence HRV, such as the reninangiotensin system, adrenomedullary catecholamines, some hormones and the direct neural innervation of the heart ${ }^{26,27,28}$.

\section{Technical approaches}

In the ambulatory setting, there are different devices to detect heartbeats, many of which, however, do not have a clinical or research application. They are based on auscultation (sound), blood pressure measurement (oscillograph), pulse oximetry (optical) and electrocardiogram (ECG). In 1996, the Task Force of the European Society of Cardiology and The North American Society of Pacing and Electrophysiology defined the methodology, standards of measurement, interpretation and clinical use of HRV using the $\mathrm{ECG}^{21}$. Based on the variation of $\mathrm{RR}$ intervals registered in a continuous span of $5 \mathrm{~min}$ to $24 \mathrm{~h}$, time-domain measures estimate those variations through different calculations. Frequency-domain measures are based on the decomposition of the total variance of RR intervals in a continuous ECG register. Initially, the timedomain and frequency-domain methods were analyzed by linear statistical methods that were explored and widely used with certain limitations in the clinical setting. Since the last decade, increased interest in the nonlinear dynamics that integrates tools from chaos theory and fractal analysis has provided novel information about the physiological mechanisms underlying HRV, with promising advances in basic and clinical applications ${ }^{29}$. 


\section{Measures}

\section{Time-domain}

Time-domain analysis evaluates the variation of successive RR intervals during a period. It requires a continuous electrocardiographic record, where each QRS complex is detected, and the normal complexes defined as resulting from normal electrical patterns are selected for analysis and called normal-to-normal (NN) intervals ${ }^{21,25}$. Two techniques are used for NN interval analysis: statistical or geometric approaches. The first uses direct measurements of NN intervals and differences between intervals, which may be calculated using smaller segments of the recording period or the whole registry. Smaller segments are useful for HRV analysis in specific activities (rest, sleep, Valsalva, deep breathing); longer registries increase NN estimates by providing more complex information ${ }^{30}$. The geometric approach is based on the NN intervals that are converted into a geometric pattern. A formula is applied to the graphic properties to measure HRV. The geometric analysis requires at least 20 minutes of monitoring. Time-domain measures recommended for HRV analysis are standard deviation of $\mathrm{NN}$ intervals (SDNN), standard deviation of the averages of $\mathrm{NN}$ intervals in all 5 min segments of the entire recording (SDANN), root square of the mean of the sum of the difference between adjacent NN intervals (RMSSD), sum of NN50 divided by the total number of NN intervals (pNN50) and the HRV triangular index ${ }^{31}$ (Table 1).

\section{Frequency-domain}

The basis of frequency-domain analysis is the spectral method. Power spectral analysis is a technique that decomposes the raw ECG waveform into its component rhythms, showing them in different frequency ranges ${ }^{32}$. The method provides information regarding power distribution as a function of frequency. Two types of methods estimate power spectral density: parametric and non-parametric. Fast Fourier transformation (FFT) is a widely used non-parametric method due to its simplicity, high processing speed, good reproducibility and low computational $\cos ^{33}$. This algorithm requires windows of 2 to $5 \mathrm{~min}$ to transform the ECG register tachogram into a representation in the frequency domain: low-frequency band, high-frequency band, very low-frequency band (Table 2).

The high-frequency band reflects parasympathetic or vagal activity and is a link to the respiratory cycle because of the respiratory sinus arrhythmia mechanism. The cardiorespiratory center inhibits vagal outflow during inspiration, with an acceleration of the HR. On the other hand, during exhalation, the HR is slowed because of vagal outflow restoration. Lower spectral power in the high-frequency range (HF) is associated with stress, panic, anxiety and reduced cognitive functions ${ }^{15,22}$. The low-frequency range primarily reflects baroreceptor activity, a reflex arc involving arterial blood pressure and heart rate. Baroreflex signals are sensed by mechanoreceptors in the heart chambers, vena cava, carotid sinuses, and the aortic arch. The vagus nerves transmit this stimulus to central structures to regulate sympathetic outflow, influencing vascular resistance

Table 1. Time-domain measurements. The measures are based on the QRS complex detected and filtered from a continuous ECG recording. The result, normal-to-normal intervals, could be analyzed with statistical or geometric approaches. The statistical method is based on direct evaluation or the differences between normal-to-normal intervals. The products from the direct evaluation are standard deviation of normal-to-normal intervals, segments of the entire recording, standard deviation of normalto-normal intervals index, and the products from the differences are sum of the difference between adjacent normal-to-normal intervals, standard deviation of the difference between adjacent normal-to-normal intervals, NN50 and total number of normalto-normal intervals.

\begin{tabular}{|c|c|c|}
\hline \multicolumn{3}{|r|}{ Statistical measures } \\
\hline SDNN & ms(milliseconds) & Standard deviation of NN intervals \\
\hline SDANN & ms(milliseconds) & Standard deviation of the averages of NN intervals in all 5 min segments of the entire recording \\
\hline RMSSD & ms(milliseconds) & Root square of the mean of the sum of the difference between adjacent NN intervals \\
\hline SDNN INDEX & ms(milliseconds) & Mean of the standard deviation of all NN intervals \\
\hline SDSD & ms(milliseconds) & Standard deviation of the difference between adjacent NN intervals \\
\hline NN50 & ms(milliseconds) & Number of adjacent NN intervals which differ more than $50 \mathrm{~ms}$ in all the registry \\
\hline pNN50 & $\%$ & Sum of NN50 divided by the total number of NN intervals \\
\hline \multicolumn{3}{|r|}{ Geometric measures } \\
\hline Triangular index HRV & & $\begin{array}{l}\text { Total number of all NN intervals divided by the height of the histogram of all NN intervals } \\
\text { (discrete scale } 1 / 128 \mathrm{~s} \text { ) }\end{array}$ \\
\hline TINN & ms(milliseconds) & $\begin{array}{c}\text { Baseline width of the minimum square difference triangular interpolation of the highest } \\
\text { peak of the histogram of all NN intervals }\end{array}$ \\
\hline Differential index HRV & ms(milliseconds) & $\begin{array}{l}\text { Difference between the widths of the histograms of the differences between adjacent } \\
\text { NN intervals measured at selected heights }\end{array}$ \\
\hline
\end{tabular}

NN: normal-to-normal; TINN: histogram of all normal-to-normal intervals; HRV: heart rate variability. 
and cardiac output according to the pressure changes sensed by the mechanoreceptors. The sympathetic nervous system does not appear to influence rhythms above $0,1 \mathrm{~Hz}$, while the parasympathetic influences heart rhythms above $0,05 \mathrm{~Hz}$. For these reasons, it has been suggested that spectral power in the low-frequency range (LF) reflects sympathetic and parasympathetic activity with a slight predominance of the first one $^{21,22,26}$. The physiological role of the very low and ultra-lowfrequency ranges are still unknown, but it is possible that they respond to the circadian rhythm, core temperature, hormones and intrinsic rhythms.

Some time- and frequency-domain variables are strongly correlated with each other. This is possible through physiological and mathematical associations. Total power is related to SDNN, HRV triangular index, and baseline width of the minimum square difference triangular interpolation of the highest peak of the histogram of all NN intervals (TINN). Spectral power in the ultra-low-frequency range (ULF) is related to SDANN. Spectral power in the high-frequency range (HF) is related to RMSSD, standard deviation of the difference between adjacent NN intervals (SDSD), number of adjacent NN intervals which differ more than $50 \mathrm{~ms}$ in all the registry (NN50) count and pNN50 $0^{21,30}$. Therefore, interpretation of the time- and frequency-domain measures together could establish a plausible, physiological and acceptable interpretation of the studied phenomenon.

\section{Nonlinear measurements}

This method is based on the principle that the underlying mechanisms of heart regulation interact in a nonlinear way ${ }^{34}$. HRV signals are non-stationary and nonlinear by definition and require for analysis dynamic methods based on chaos theory and nonlinear system theory. Nonlinear methods are not based on prespecified patterns and could use measures based on the regularity of signals (entropy) and self-similarities within signals (fractal methods). Approximate entropy (ApEN), a measure of repeated structures, is inversely proportional to regularity, with lower values indicating higher regularity. Sample entropy (SampEn) estimates the probability of identifying specific patterns in a time series; values near two represent complete chaos. In clinical studies, the significance os these measurements as prognostic tool is still unclear ${ }^{35}$.

Fractal analysis is used to find temporal self-similarities over a range of scales. Detrended fluctuation analysis (DFA) needs 1,000 beats to analyze the statistical self-affinity of signals. Lower values are associated with random series and high values indicate a time-series correlation. Other methods used in fractal analysis are Fano factor, dispersion analysis, fractal dimension, correlation dimension and coarse-grained spectral analysis, all of which have unknown application in the clinical setting ${ }^{36}$.

\section{HEART RATE VARIABILITY AND STROKE}

Growing evidence suggests that the pathophysiological process during the acute phase of stroke is not an isolated brain process. Inflammatory, endocrine and autonomic pathways are activated in parallel to the ischemic cascade with systemic responses ${ }^{37,38}$. Unfortunately, the majority of responses have harmful effects clinically manifested as infection, fever, delirium, thrombosis, metabolic disturbances, and cardiac complications $\mathrm{s}^{39}$. There is some concern about the persistence of dysfunctions over time and their long-term

Table 2. Frequency-domain measurements. Short-term registries are recorded in 2 to 5 minutes and are the basis for the longterm registries. The 24-hour registries are usually divided into "windows" of 5 minutes for the analysis, that could be a specific Hanning window or an average of all the windows. The selection of a specific window depends on the aim of the research and the studied phenomenon.

\begin{tabular}{|c|c|c|c|}
\hline Variable & Units & Description & Frequency range \\
\hline \multicolumn{4}{|c|}{ Short-term registry (5 minutes) } \\
\hline 5 minute registry & ms2 & Variance of the NN intervals in the time segment & $<0.4 \mathrm{~Hz}$ \\
\hline VLF & ms2 & Spectral power in the very low-frequency range & $<0.04 \mathrm{~Hz}$ \\
\hline LF & ms2 & Spectral power in the low-frequency range & $0.04-0.14 \mathrm{~Hz}$ \\
\hline LF normalized & n.u & Normalized spectral power in the low-frequency range/(total power-VLF)×100 & - \\
\hline $\mathrm{HF}$ & ms2 & Spectral power in the high-frequency range & $0.25-0.4 \mathrm{~Hz}$ \\
\hline LF/HF & & Frequency index & \\
\hline \multicolumn{4}{|c|}{ Long-term registry (24 hours) } \\
\hline Long-term registry & & Variance of all NN intervals & $<0.4 \mathrm{~Hz}$ \\
\hline ULF & ms2 & Spectral power in the ultra-low-frequency range & $<0.003 \mathrm{~Hz}$ \\
\hline VLF & ms2 & Spectral power in the very low-frequency range & $0.003-0.04 \mathrm{~Hz}$ \\
\hline LF & ms2 & Spectral power in the low-frequency range & $0.04-0.15 \mathrm{~Hz}$ \\
\hline $\mathrm{HF}$ & ms2 & Spectral power in the high-frequency range & $0.15-0.4 \mathrm{~Hz}$ \\
\hline
\end{tabular}


consequences for stroke patients, with an impact on morbidity, mortality, and quality of life ${ }^{40}$.

The ANS shows altered responses in the acute and chronic phases of IS. During the acute phase, patients exhibit higher catecholamines levels compared to controls, as well as fever and arrhythmias. Stroke-heart syndrome includes cardiac manifestations induced by IS after the onset of the neurological deficits, and represents a medical challenge during stroke care $^{41,42}$. Clinical manifestations vary from asymptomatic ECG alterations to malignant cardiac arrhythmia, worsening of left ventricular function and stroke-induced myocardial infarction. The autonomic cardiac control studied by HRV analysis in stroke patients suggests different physiological states or responses to pathological conditions that could be used for prognosis and etiology definition, establishing future therapeutic targets.

\section{Technical issues of heart rate variability in stroke}

The recommendations for measures and techniques of HRV in patients with stroke are the same as for other diseases described by the Task Force for measurements and standards of HRV but there is great diversity and heterogeneity of methods applied in the studies reported in the literature. In the acute phase, patients are usually in an in-hospital setting with several factors, such as medications, stress, airway support and sedation, that could influence HRV analysis. In the chronic phase patients are in an ambulatory context and the influence of other factors such as medications (betablockers, antiarrhythmics, sedatives), functional dependency (prolonged disability), sleep disorders and multiple comorbidities that have to considered for interpretation of HRV analysis. Stroke patients by definition are a high-risk cardiovascular population with a high prevalence of associated diseases, such as atrial fibrillation, heart failure, arrhythmias, and diabetic neuropathy, conditions that can interfere with HRV measures. Finally, it is important to note that the effects of stroke in ACN and ANS functions are reflected in HRV parameters and override the physiological effects of aging and sex in HRV analysis ${ }^{43}$.

\section{Acute phase}

HRV could be used in the acute phase of stroke to predict the risk of mortality, morbidity and possible complications. Studies evaluating patients in the acute phase of stroke showed that HRV time-domain measures (SDNN, SDANN, RMSS) are lower and frequency-domain measures (spectral power in the low-frequency range $-\mathrm{LF}$, frequency index - LF/HF) exhibit low-frequency predominance in sympathetic to parasympathetic swings compared to healthy controls. Also, baroreceptor reflex sensitivity is impaired and a sympathetic predominance is observed three days after stroke ${ }^{44}$. These alterations are linked to a higher risk of mortality, sudden cardiac death and hypertensive crises probably related to autonomic dysfunction during the circadian cycle ${ }^{45}$. The heart-brain axis, disturbed in stroke patients, is manifested with ECG abnormalities that may be present in more than $20 \%$ of them. The topography of the ischemic lesion is related to autonomic disturbances and specific arrhythmogenic patterns that are seen especially in the insular cortex lesions ${ }^{43,46}$. Neurogenic atrial fibrillation is an example of an autonomic imbalance in the context of insular cortex ischemia, a phenomenon that is more severe during the acute phase and tends to disappear during the first month after stroke ${ }^{47}$.

\section{Chronic phase}

HRV analysis in the chronic phase could be useful to predict recurrence, mortality and possible etiological mechanisms. Fewer studies are available evaluating long-term changes in HRV because it is presumed that the autonomic effects of stroke disappear with time. Some studies have demonstrated that autonomic dysfunction in stroke patients could be prolonged for up to four years after the ictus and is manifested as reduced parasympathetic activity ${ }^{48,49}$. Also, recent studies showed that abnormal time-domain (SDNN, RMSS), frequency-domain (LF, HF, spectral power in the very low-frequency range - VLF) parameters and nonlinear entropy parameters (DFA, ApEN) are predictors for stroke severity, functional outcome, and mortality ${ }^{50}$. Less variability, loss of parasympathetic tonus and HRV parameters altered during the sleep cycle are associated with worse prognosis $^{50,51}$. However, HRV parameters as biomarkers for stroke still require the integration of linear and nonlinear methods to expand the risk modeling and improve its predictive value.

\section{DISCUSSION}

Stroke is a devastating disease with great impact in different scenarios. Autonomic dysfunction is frequently observed in patients with IS in the acute and chronic stages of the disease. $\mathrm{HRV}$ analysis as an approach for testing autonomic function is a noninvasive method with growing application in the clinical setting. IS patients show decreased HRV and an imbalance in the sympathetic/parasympathetic tonus that persists over time. It is known that altered parameters of HRV are independent predictors of mortality and functional outcome ${ }^{52}$. The pathophysiological mechanisms of cardiovascular autonomic dysfunction in IS patients remain unclear, but a better understanding of HRV methods could help to resolve these questions.

With the epidemiological transition, chronic diseases are a particular concern. Stroke is one of the leading causes of mortality and disability worldwide, so the development of preventive and therapeutic strategies should be a high priority. Since the last decade, great advances in reperfusion therapies have been changing the classical history of stroke patients. However, the vast majority still experiences the serious consequences of having an I $^{53,54}$. Indeed, efforts in research must be guided to find the best strategies for the prevention and care of stroke patients. 
Autonomic disturbances are common after stroke and are usually misunderstood and not well studied. One explanation for this is the complexity of the ANS, which complicates the study of isolated effects of specific pathways due to the hierarchical, intricate and multiple set-points inside the system. Also, ANS evaluation in the clinical setting is limited because tests have been designed for research purposes, having high costs and low availability. Confounding factors such as comorbidities, medication use, state of hydration and prolonged severe disability could alter results. Even though autonomic tests are non-invasive and easy to apply, they assess effector responses and end-organ failure by itself can alter the results. On the other hand, during the acute phase of IS the treatment objectives are directed to the reperfusion, care and stabilization of the patient and the role of autonomic disturbances are relegated to a secondary or tertiary endpoint. In the chronic phase, secondary prevention, rehabilitation, and etiology determination are the main objectives, and the role of autonomic dysfunction in this context is not well studied.

Heart rate variability parameters in IS patients as a cardiac autonomic biomarker is correlated with prognosis. The altered linear measurements showed associations with stroke severity, early and late complications and mortality. The role of nonlinear methods as a prognostic tool or for the understanding of autonomic disturbances in IS patients is still a topic of debate. Studies applying the standards of measurement for HRV analysis with uniform endpoints, adequate sample size and integrating nonlinear methods are required to elucidate the factors influencing HRV control in IS patients.

\section{CONCLUSIONS}

Autonomic disturbances after IS are common and cardiac complications during the acute phase are challenging conditions. HRV analysis in IS patients is a valuable approach to understand physiopathological mechanisms and could be used as a prognostic tool.

HRV parameters are altered after IS. Patients exhibit less variability and a shift to sustained sympathetic tonus during the circadian cycle, with reduced parasympathetic activity that seems to persist until the chronic stage of the disease.

For future research it is necessary to harmonize and integrate linear and nonlinear methods of HRV analysis to obtain better prognostic value and to understand the heart and brain interactions that influence HRV control.

\section{References}

1. Ministério da Saúde do Brasil. Análise situação em saúde e desafios nos Objetivos do Desenvolvimento Sustentável. Brasília: Secretaria de Vigilância da Saúde; 2017. Available from: http://bvsms.saude.gov. br/bvs/publicacoes/saude_brasil_2017_analise_situacao_saude_ desafios_objetivos_desenvolvimento_sustetantavel.pdf.

2. Katan M, Luft A. Global burden of stroke. Semin Neurol. 2018 Apr;38(2):208-11. https://doi.org/10.1055/s-0038-1649503

3. Brouns R, De Deyn PP. The complexity of neurobiological processes in acute ischemic stroke. Clin Neurol Neurosurg. 2009 Jul;111(6):48395. https://doi.org/10.1016/j.clineuro.2009.04.001

4. Benarroch EE. The central autonomic network: functional organization, dysfunction, and perspective. Mayo Clin Proc. 1993 Oct;68(10):988-1001. https://doi.org/10.1016/s0025-6196(12)62272-1

5. Manea MM, Comsa M, Minca A, Dragos D, Popa C. Brain-heart axisReview Article. J Med Life. Jul-Sep 2015;8(3):266-71.

6. Daniele O, Caravaglios G, Fierro B, Natalè E. Stroke and cardiac arrhythmias. J Stroke Cerebrovasc Dis. Jan-Feb 2002;11(1):28-33. https://doi.org/10.1053/jscd.2002.123972

7. Freeman R, Chapleau MW. Testing the autonomic nervous system. Handb Clin Neurol. 2013;115:115-36. https://doi.org/10.1016/B9780-444-52902-2.00007-2

8. Seifert F, Kallmünzer B, Gutjahr I, Breuer L, Winder K, Kaschka I, et al. Neuroanatomical correlates of severe cardiac arrhythmias in acute ischemic stroke. J Neurol. 2015 May;262(5):1182-90. https://doi. org/10.1007/s00415-015-7684-9

9. Ruthirago D, Julayanont P, Tantrachoti P, Kim J, Nugent K. Cardiac arrhythmias and abnormal electrocardiograms after acute stroke. Am J Med Sci. 2016 Jan;351(1):112-8. https://doi.org/10.1016/j. amjms.2015.10.020

10. Wehrwein EA, Orer HS, Barman SM. Overview of the anatomy, physiology, and pharmacology of the autonomic nervous system. Compr Physiol. 2016 Jun;6(3):1239-78. https://doi.org/10.1002/cphy.c15003
11. Cannon WB. the interrelations of emotions as suggested by recent physiological researches. Am J Psychol. 1914 Apr;25(2):256-82. https://doi.org/10.2307/1413414

12. Hall JE, Guyton AC. Guyton and Hall textbook of medical physiology. 12. ed. Philadelphia, PA: Saunders/Elsevier; 2011.

13. Beissner F, Meissner K, Bär K-J, Napadow V. The autonomic brain: an activation likelihood estimation meta-analysis for central processing of autonomic function. J Neurosci. 2013 Jun 19;33(25):10503-11. https://doi.org/10.1523/JNEUROSCI.1103-13.2013

14. Sklerov M, Dayan E, Browner N. Functional neuroimaging of the central autonomic network: recent developments and clinical implications. Clin Auton Res. 2019 Dec;29(6):555-66. https://doi. org/10.1007/s10286-018-0577-0

15. Karemaker JM. An introduction into autonomic nervous function. Physiol Meas. 2017 May;38(5):R89-R118. https://doi. org/10.1088/1361-6579/aa6782

16. Ziemssen T, Siepmann T. The investigation of the cardiovascular and sudomotor autonomic nervous system - a review. Front Neurol. 2019 Feb;10:53. https://doi.org/10.3389/fneur.2019.00053

17. Sletten DM, Suarez GA, Low PA, Mandrekar J, Singer W. COMPASS 31: a refined and abbreviated composite autonomic symptom score. Mayo Clin Proc. 2012 Dec;87(12):1196-201. https://doi.org/10.1016/j. mayocp.2012.10.013

18. Macey PM, Ogren JA, Kumar R, Harper RM. Functional imaging of autonomic regulation: methods and key findings. Front Neurosci. 2016 Jan;9:513. https://doi.org/10.3389/fnins.2015.00513

19. Boylan M. Galen: on blood, the pulse, and the arteries. J Hist Biol. Summer 2007;40(2):207-30. https://doi.org/10.1007/s10739-006-9116-2

20. AlGhatrif M, Lindsay J. A brief review: history to understand fundamentals of electrocardiography.J Community Hosp Intern Med Perspect. 2012 Apr 30;2(1). https://doi.org/10.3402/jchimp. v2i1.14383 
21. Task Force. Heart rate variability. Standards of measurement, physiological interpretation, and clinical use. Task Force of The European Society of Cardiology and the North American Society for Pacing and Electrophysiology. Eur Heart J. 1996;17(3):354-81.

22. Cygankiewicz I, Zareba W. Heart rate variability. Handb Clin Neurol. 2013;117:379-93. https://doi.org/10.1016/B978-0-444-53491$0.00031-6$

23. Moorman JR, Delos JB, Flower AA, Cao H, Kovatchev BP, Richman JS, et al. Cardiovascular oscillations at the bedside: early diagnosis of neonatal sepsis using heart rate characteristics monitoring. Physiol Meas. 2011 Nov;32(11):1821-32. https://doi.org/10.1088/09673334/32/11/S08

24. Fairchild KD, Schelonka RL, Kaufman DA, Carlo WA, Kattwinkel $\mathrm{J}$, Porcelli PJ, et al. Septicemia mortality reduction in neonates in a heart rate characteristics monitoring trial. Pediatr Res. 2013 Nov;74(5):570-5. https://doi.org/10.1038/pr.2013.136

25. Singh N, Moneghetti KJ, Christle JW, Hadley D, Plews D, Froelicher V. Heart rate variability: an old metric with new meaning in the era of using mHealth technologies for health and exercise training guidance. Part One: Physiology and Methods. Arrhythm Electrophysiol Rev. 2018 Aug;7(3):193-8. https://doi.org/10.15420/aer.2018.27.2

26. Gordan R, Gwathmey JK, Xie L-H. Autonomic and endocrine control of cardiovascular function. World J Cardiol. 2015 Apr;7(4):204-14. https://doi.org/10.4330/wjc.v7.i4.204

27. Mendelowitz D. Advances in parasympathetic control of heart rate and cardiac function. News Physiol Sci. 1999 Aug;14:155-61. https:// doi.org/10.1152/physiologyonline.1999.14.4.155

28. Kumar KR, Kirsch RE, Hornik CP. Cardiovascular physiology for intensivists. In: Critical heart disease in infants and children. 3. ed. Elsevier; 2019. p. 111-133.e5. Available from: https://linkinghub. elsevier.com/retrieve/pii/B9781455707607000139

29. Sassi R, Cerutti S, Lombardi F, Malik M, Huikuri HV, Peng C-K, et al. Advances in heart rate variability signal analysis: joint position statement by the e-Cardiology ESC Working Group and the European Heart Rhythm Association co-endorsed by the Asia Pacific Heart Rhythm Society. Europace. 2015 Sep;17(9):1341-53. https://doi. org/10.1093/europace/euv015

30. Trimmel K, Sacha J, Huikuri HV, editors. Heart rate variability: clinical applications and interaction between HRV and heart rate. Frontiers Media SA; 2015. (Frontiers Research Topics). Available from: http:// www.frontiersin.org/books/Heart_Rate_Variability_Clinical_ Applications_and_Interaction_between_HRV_and_Heart_Rate/698

31. Dutsch M, Burger M, Dorfler C, Schwab S, Hilz MJ. Cardiovascular autonomic function in poststroke patients. Neurology. 2007 Dec;69(24):2249-55. https://doi.org/10.1212/01. wnl.0000286946.06639.a7

32. McCraty R, Shaffer F. Heart rate variability: new perspectives on physiological mechanisms, assessment of self-regulatory capacity, and health risk. Glob Adv Health Med. 2015 Jan;4(1):46-61. https:// doi.org/10.7453/gahmj.2014.073

33. Li K, Rüdiger H, Ziemssen T. Spectral analysis of heart rate variability: time window matters. Front Neurol. 2019 May;10:545. https://doi. org/10.3389/fneur.2019.00545

34. Germán-Salló Z, Germán-Salló M. Non-linear methods in HRV analysis. Procedia Technol. 2016;22:645-51. https://doi. org/10.1016/j.protcy.2016.01.134

35. Ernst G. Hidden signals — the history and methods of heart rate variability. Front Public Health. 2017 Oct;5:265. https://doi. org/10.3389/fpubh.2017.00265

36. Shaffer F, Ginsberg JP. An overview of heart rate variability metrics and norms. Front Public Health. 2017;5:258. https://doi.org/10.3389/ fpubh.2017.00258

37. El Husseini N, Laskowitz DT. The role of neuroendocrine pathways in prognosis after stroke. Expert Rev Neurother. 2014 Feb;14(2):217-32. https://doi.org/10.1586/14737175.2014.877841
38. Fu Y, Liu Q, Anrather J, Shi F-D. Immune interventions in stroke. Nat Rev Neurol. 2015 Sep;11(9):524-35. https://doi.org/10.1038/ nrneurol.2015.144

39. Kumar S, Selim MH, Caplan LR. Medical complications after stroke. Lancet Neurol. 2010 Jan;9(1):105-18. https://doi.org/10.1016/S14744422(09)70266-2

40. Maaijwee NA, Rutten-Jacobs LC, Schaapsmeerders P, van Dijk EJ, de Leeuw F-E. Ischaemic stroke in young adults: risk factors and longterm consequences. Nat Rev Neurol. 2014 Jun;10(6):315-25. https:// doi.org/10.1038/nrneurol.2014.72

41. Scheitz JF, Nolte CH, Doehner W, Hachinski V, Endres M. Strokeheart syndrome: clinical presentation and underlying mechanisms. Lancet Neurol. 2018 Dec;17(12):1109-20. https://doi.org/10.1016/ S1474-4422(18)30336-3

42. Chen Z, Venkat P, Seyfried D, Chopp M, Yan T, Chen J. Brain-heart interaction: cardiac complications after stroke. Circ Res. 2017 Aug;121(4):451-68. https://doi.org/10.1161/CIRCRESAHA.117.311170

43. Dorrance AM, Fink G. Effects of stroke on the autonomic nervous system. In:Terjung R, editor. Comprehensive physiology. Hoboken, NJ, USA: John Wiley \& Sons, Inc.; 2015. p. 1241-63. Available from: http:// doi.wiley.com/10.1002/cphy.c140016

44. Yperzeele L, van Hooff R-J, Nagels G, De Smedt A, De Keyser J, Brouns R. Heart rate variability and baroreceptor sensitivity in acute stroke: a systematic review. Int J Stroke. 2015 Aug;10(6):796-800. https://doi.org/10.1111/ijs.12573

45. Palamarchuk I, Kimpinski K, Lippert C, Hachinski V. Nocturnal deterioration after ischemic stroke and autonomic dysfunction: hypothesis and implications. Cerebrovasc Dis. 2013;36(5-6):454-61. https://doi.org/10.1159/000356093

46. Raghu ALB, Parker T, van Wyk A, Green AL. Insula stroke: the weird and the worrisome. Postgrad Med J. 2019 Sep;95(1127):497-504. http://dx.doi.org/10.1136/postgradmedj-2019-136732

47. Sposato LA, Riccio PM, Hachinski V. Poststroke atrial fibrillation: cause or consequence? Critical review of current views. Neurology. 2014 Apr;82(13):1180-6. https://doi.org/10.1212/ WNL.0000000000000265

48. Grilletti J, Scapini K, Bernardes N, Spadari J, Bigongiari A, Mazuchi $F$, et al. Impaired baroreflex sensitivity and increased systolic blood pressure variability in chronic post-ischemic stroke. Clinics. 2018 Oct;73:e253. https://doi.org/10.6061/clinics/2018/e253

49. Lees T, Shad-Kaneez F, Simpson AM, Nassif NT, Lin Y, Lal $\mathrm{S}$. Heart rate variability as a biomarker for predicting stroke, post-stroke complications and functionality. Biomark Insights. 2018;13:1177271918786931. https://doi. org/10.1177/1177271918786931

50. Tobaldini E, Proserpio P, Oppo V, Figorilli M, Fiorelli EM, Manconi M, et al. Cardiac autonomic dynamics during sleep are lost in patients with TIA and stroke. J Sleep Res. 2019 Jun;29(3):e12878. https://doi. org/10.1111/jsr.12878

51. Binici Z, Mouridsen MR, Køber L, Sajadieh A. Decreased nighttime heart rate variability is associated with increased stroke risk. Stroke. 2011 Nov;42(11):3196-201. https://doi.org/10.1161/ STROKEAHA.110.607697

52. Oka H. Heart rate variability and neurological disorders. In: Iwase S, Hayano J, Orimo S, editors. Clinical assessment of the autonomic nervous system. Tokyo: Springer Japan; 2017. p.179-97. Available from: http://link.springer.com/10.1007/978-4-43156012-8_11

53. Pandian JD, Gall SL, Kate MP, Silva GS, Akinyemi RO, Ovbiagele $\mathrm{BI}$, et al. Prevention of stroke: a global perspective. Lancet. 2018 Oct;392(10154):1269-78. https://doi.org/10.1016/S01406736(18)31269-8

54. Ghandehari K. Barriers of thrombolysis therapy in developing countries. Stroke Res Treat. 2011;2011:686797. https://doi. org/10.4061/2011/686797 